\title{
Sobre el diagnóstico y tratamiento de la displasia quística de la vesícula seminal
}

\author{
P. Navalón Verdejo,**,***, Y. Pallas Costa*, J.A. Cánovas Ivorra*, F. Ordoño Domínguez**, \\ L. de la Torre Abril**, M. Fabuel Deltoro** \\ *Servicio de Urología. Hospital Casa de Salud. Valencia. \\ **Servicio de Urología. Consorcio Hospital General Universitario. Valencia. \\ ***Departamento de Cirugía. Facultad de Medicina. Universidad de Valencia.
}

Actas Urol Esp 2006; 30 (2): 152-158

\section{RESUMEN}

SOBRE EL DIAGNÓSTICO Y TRATAMIENTO DE LA DISPLASIA QUÍSTICA DE LA VESÍCULA SEMINAL

Introducción y objetivo: La displasia quística de la vesícula seminal representa una rara anomalía congénita urológica cuyo tratamiento de elección es controvertido. Presentamos nuestra experiencia en el diagnóstico y tratamiento de esta infrecuente entidad.

Pacientes y método: Durante los últimos 12 años hemos atendido a 8 pacientes afectos de esta patología. En 3 de ellos, asintomáticos, el diagnóstico fue incidental, mientras que los 5 restantes consultaron por síntomas del tracto urinario inferior. En 3 pacientes se halló desembocadura ectópica de un uréter remanente en la vesícula patológica, 2 de ellos con importante ectasia renoureteral. Los otros 5 pacientes presentaron agenesia renal.

Resultados: En los pacientes asintomáticos adoptamos una actitud expectante, optando por la cirugía en los otros 5. Realizamos cirugía exerética en los 2 casos que presentaban ureterohidronefrosis, mientras que en los otros 3 realizamos incisión endoscópica de la vesícula seminal, obteniendo excelentes resultados en todos ellos, sin evidenciarse aparición de pseudodivertículo sintomático.

Conclusiones: La incisión transuretral endoscópica del quiste de la vesícula seminal es un procedimiento mínimamente invasivo y con escasa morbilidad, habiendo constatado la retracción prácticamente completa del quiste en todos nuestros casos, por lo que la consideramos de elección para el tratamiento de los pacientes afectos de dicha entidad, reservando la cirugía abierta para los casos en los que fracase la técnica por aparición de un pseudodivertículo sintomático y en los pacientes que presenten asociada ectasia del remanente renoureteral.

Palabras clave: Quiste de vesícula seminal. Agenesia renal. Tratamiento quirúrgico.

\section{ABSTRACT}

DIAGNOSIS AND MANAGEMENT OF CYSTIC DYSPLASIA OF THE SEMINAL VESICLE

Introduction and objectives: Cystic dysplasia of the seminal vesicle represents a rare congenital urologic anomaly of which elective treatment is controversial. We present our experience in the diagnosis and management of this uncommon pathology.

Patients and method: During the last twelve years we have managed eight patients affected by this pathology. The diagnosis was incidental in the three asymptomatic patients, while the other five consulted because of low urinary tract sympthoms. Ectopic drainage of the ureter in the pathological seminal vesicle was found in three of the patients. Two of these patients presented large pelvic and ureteral ectasy. Other five patients presented renal agenesis.

Results: In asymptomatic patients we adopted an expectant attitude, while the other five patients underwent surgical treatment. We performed open surgery in the two cases presenting ureterohydronephrosis, whereas in the other three we performed transurethral incision of the seminal vesicle. We obtained excellent results in all of them, with no evidence of symptomatic pseudodiverticulum.

Conclusions: Transurethral incision of the seminal vesicle cyst is a minimal invasive procedure with low morbidity. We have proved in all of our cases that the retraction of the cyst was practically complete, this has led us into believing that transurethral incision is the elective treatment. Therefore, open surgery would only be indicated in cases of failure of the procedure due to existence of symptomatic pseudodiverticulum, or in patients presenting renoureteral ectasy associated.

Keywords: Seminal vesicle cysts. Renal agenesis. Surgical treatment. 
$\mathrm{L}$ os quistes de la vesícula seminal constituyen una anomalía congénita urológica de infrecuente aparición, que junto con los quistes del conducto mulleriano, los del utrículo prostático, las dilataciones quísticas del conducto eyaculador y ampolla deferencial y los quistes prostáticos, forman parte de los denominados quistes pélvicos profundos ${ }^{1}$.

Hasta hace unas décadas, la forma de diagnóstico más frecuentemente de esta entidad la constituía su hallazgo casual durante la autopsia, ya que usualmente no producen manifestaciones clínicas relevantes, siendo aún en la actualidad muchas veces diagnosticadas de forma incidental durante el estudio de otra patología, pero desde la aparición de la ecografía y particularmente desde que se popularizó la ecografia transrectal, su incidencia ha aumentado, aunque sigue tratándose de una patología de rara presentación. Así, Sheih et al. ${ }^{2}$ establecen su frecuencia en 0,002\% tras una búsqueda ultrasonográfica en 280.000 niños y adolescentes, siendo la forma de presentación bilateral un hallazgo excepcional.

En aproximadamente las dos terceras partes de los casos, los quistes de vesícula seminal se acompañan de agenesia renal ipsilateral ${ }^{1,3}$. En ocasiones existe un abocamiento ectópico del uréter en vesículas seminales con displasia quística asociada a displasia o agenesia renal ${ }^{4}$. También se han publicado casos asociados a enfermedad poliquística renal del adulto, en cuya patogénesis algunos autores sugieren que pudiera deberse a un defecto general de la membrana basal de varios órganos, entre ellos las vesículas seminales ${ }^{5}$.

El anormal desarrollo embriológico que produce esta anomalía está todavía por esclarecer en toda su extensión. No obstante, todos los autores consultados coinciden en que una anomalía primaria del conducto mesonéfrico es la causa de esta infrecuente malformación.

\section{MATERIAL Y MÉTODOS}

Pacientes y manifestaciones clinicas

Durante los últimos 12 años, hemos tenido la oportunidad de diagnosticar y tratar a 8 pacientes afectos de esta anomalía. En tres de ellos, asintomáticos, con edades comprendidas entre
51 y 68 años, el diagnóstico se efectuó incidentalmente durante la práctica de una biopsia prostática transrectal ecodirigida por elevación sérica de PSA. Los cinco pacientes restantes, con edades comprendidas entre 17 y 25 años (media: 23,4 años), presentaron diversa sintomatología genitourinaria, que varió desde hemospermia en un caso, epididimitis de repetición en dos casos y molestias abdómino-perineales inespecíficas en los otros dos.

\section{Métodos diagnósticos}

La ultrasonografía abdominal demostró en todos los casos la presencia de una imagen hipoecogénica retrovesical lateral (Fig. 1) así como la existencia de uréterohidronefrosis con parénquima renal inapreciable en dos pacientes (en ambos casos en el lado izquierdo), no hallándose en los otros 6 casos restantes imágenes sugestivas de unidad renal en uno de los lados, siendo la relación derecho/izquierdo de 2/4.

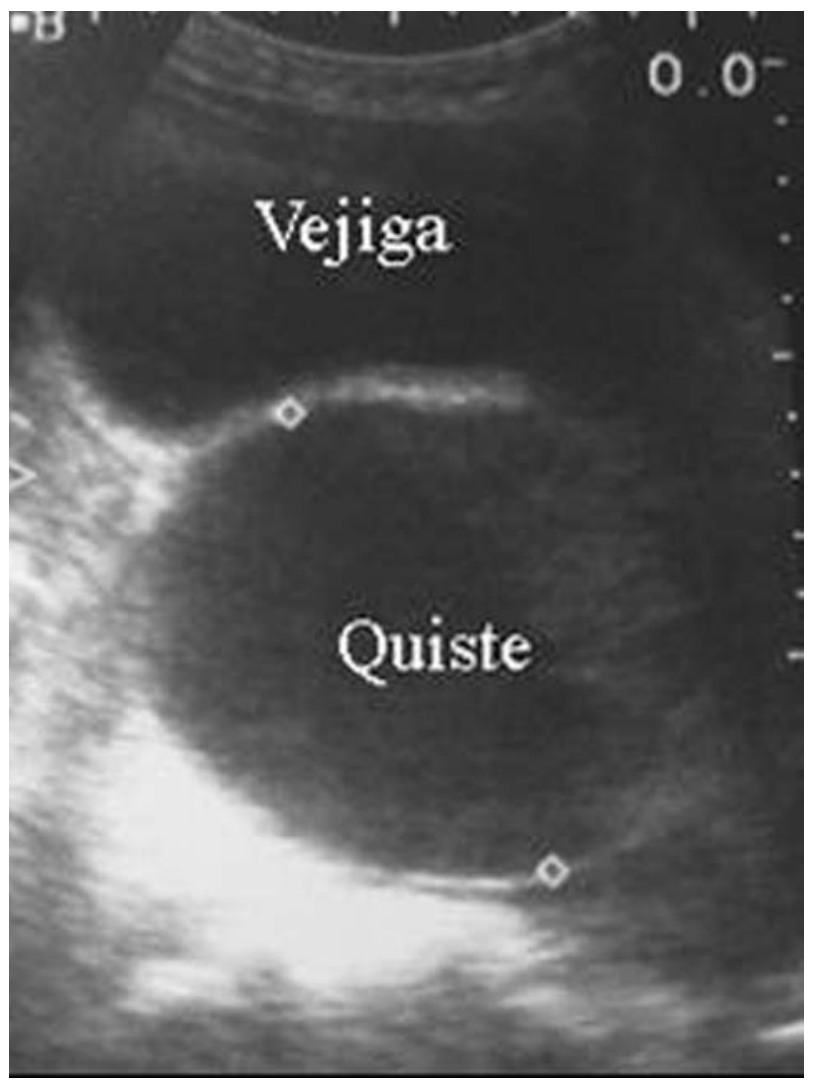

FIGURA 1. Imagen ecográfica abdominal del quiste de vesícula seminal. 
La ecografía transrectal, practicada a 6 de los pacientes comprobó la existencia de una estructura hipoecogénica retrovesical y lateroprostática.

El estudio urográfico, que se realizó en 5 pacientes, puso de manifiesto la anulación funcional de una unidad renal, evidenciándose además en la cistografía el defecto de repleción ocasionado por la compresión vesical de la vesícula seminal quística en 3 casos.

Solamente en uno de los pacientes que presentaban ureterohidronefrosis realizamos quistografía retrógrada por punción, comprobando el abocamiento ectópico del uréter en la vesícula seminal quística (Figura 2). Asimismo, en este caso, el análisis del líquido aspirado evidenció la presencia de espermatozoides.

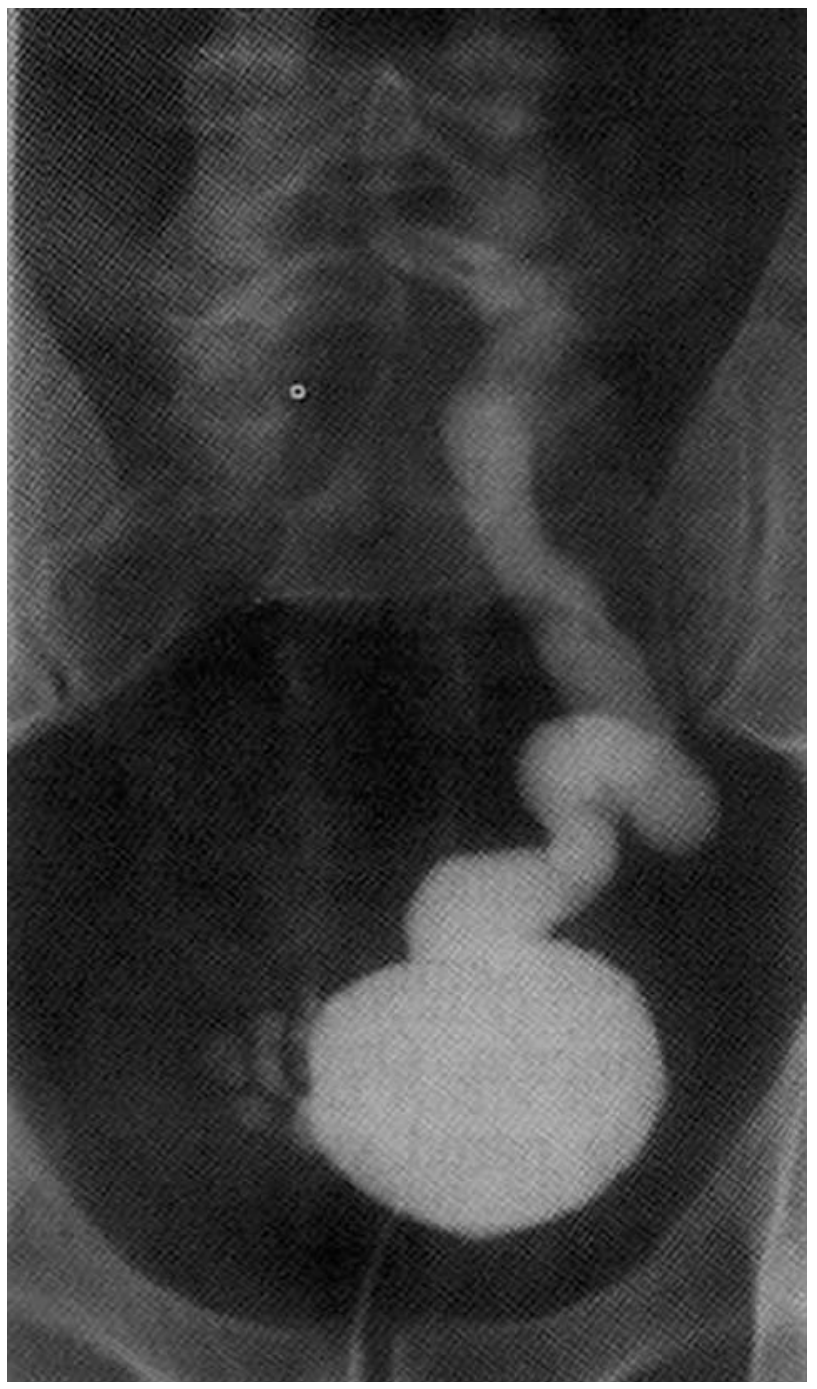

FIGURA 2. Imagen de quistografia donde se observa el relle no del quiste, la vesícula seminal y el uréter remanente.
Tanto el estudio TC realizado en 5 casos como la RM practicada en los otros 3 , evidenciaron la dilatación quística de la vesícula seminal (Figs. 3 y 4), poniendo también de manifiesto la coexistencia de agenesia renal unilateral en 5 pacientes, la ureterohidronefrosis en 2 casos así como la presencia en otro paciente de un riñón hipoplásico ipsilateral con uréter atrófico que pasó

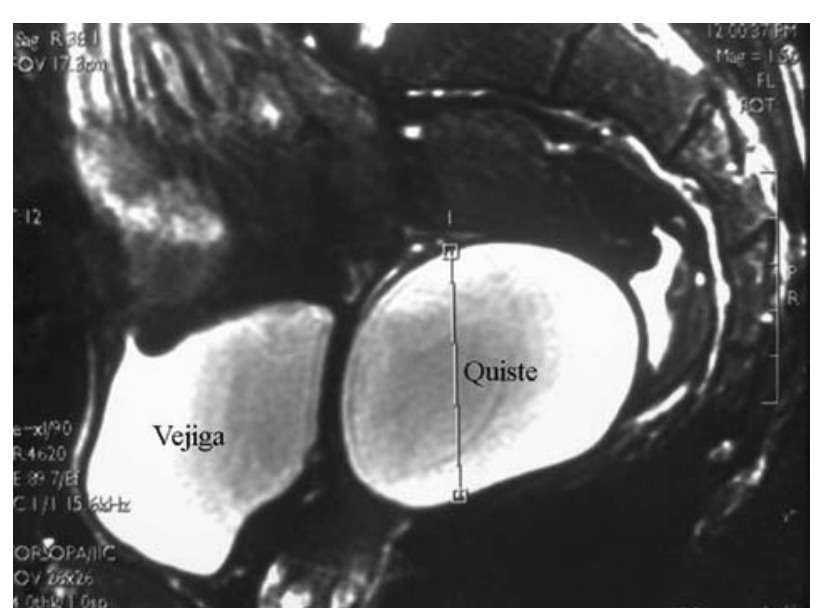

FIGURA 3. Imagen RM de quiste de vesícula seminal.

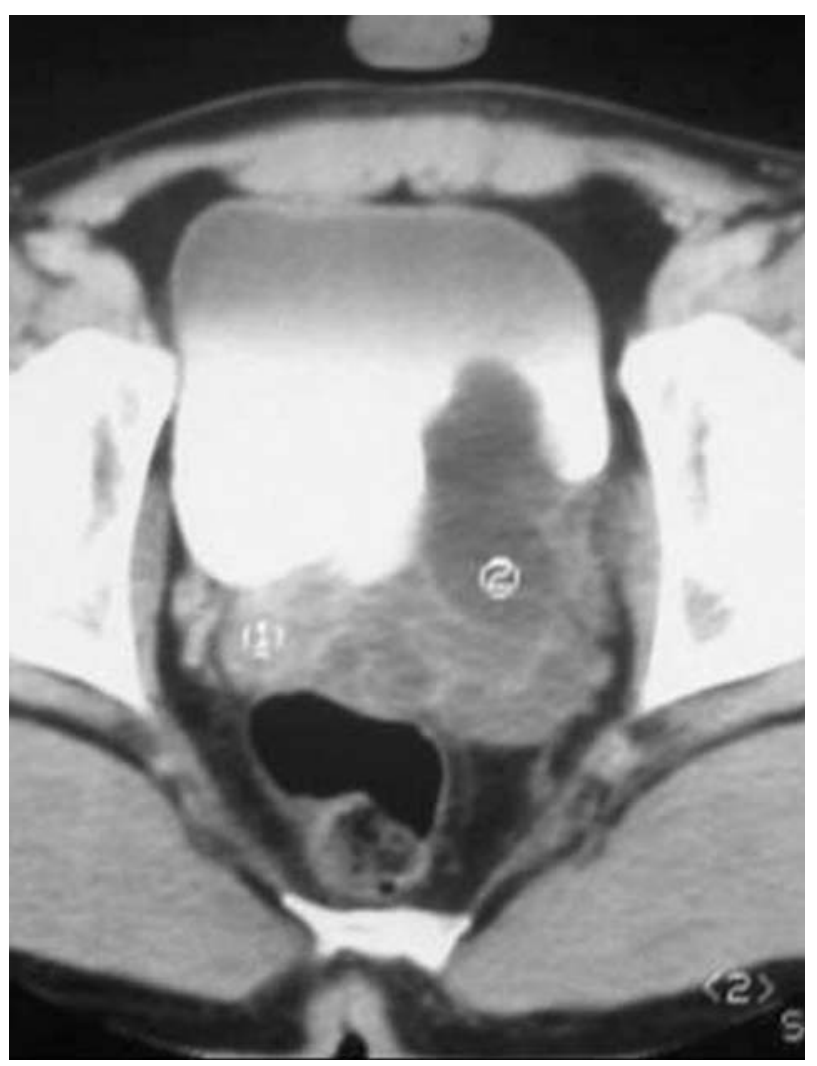

FIGURA 4. Imagen TC de quiste de vesicula seminal. 
desapercibido en el estudio por ultrasonidos y urográfico debido a su escaso tamaño y ausencia de función.

La cistoscopia, realizada a 4 pacientes demostró la ausencia del hemitrígono correspondiente así como la compresión vesical posterolateral provocada por la vesícula seminal patológica.

\section{Tratamientos realizados}

La abstención quirúrgica y la observación periódica constituyeron la única indicación en los tres pacientes asintomáticos que fueron diagnosticados incidentalmente.

En los 5 pacientes que presentaban sintomatología indicamos una actitud quirúrgica. Así, en los 2 casos que presentaban ureterohidronefrosis realizamos exéresis del remanente renoureteral ectásico y de la vesícula seminal patológica utilizando un abordaje retroperitoneal a través de una incisión de Gibson, siendo el estudio anatomopatológico informado como ureteritis erosiva acompañada de restos metanéfricos paraureterales en ambos casos.

En los otros 3 casos restantes que no presentaban ureterohidronefrosis, optamos por realizar tratamiento mínimamente invasivo mediante la incisión endoscópica transuretral de la vesícula seminal con asa de Collins drenando el contenido del quiste al interior de la vejiga.

\section{RESULTADOS}

Los 3 pacientes asintomáticos, que fueron diagnosticados incidentalmente, no han presentado variaciones clínicas ni ecográficas tras un seguimiento medio de 53 meses (rango 32-65 meses).

Los 2 pacientes a los que se realizó nefroureterectomía, el tiempo quirúrgico medio fue de aproximadamente 150 minutos, precisando una estancia postoperatoria de 12 y 15 días. En ambos la evolución fue satisfactoria, siendo dados de alta tras cinco años de seguimiento.

En los 3 pacientes que fueron tratados endoscópicamente mediante incisión transuretral del quiste y drenaje vesical del contenido del mismo, la duración del acto quirúrgico fue en todos los casos inferior a 15 minutos, siendo dados de alta hospitalaria sin sonda uretrovesical a las 24 horas del postoperatorio. En todos los casos hemos constatado, mediante estudio TC realizado postoperatoriamente, la retracción prácticamente completa de la vesícula seminal (Fig. 5) así como la desaparición de los síntomas tras un seguimiento medio de 28 meses (rango 12-46).

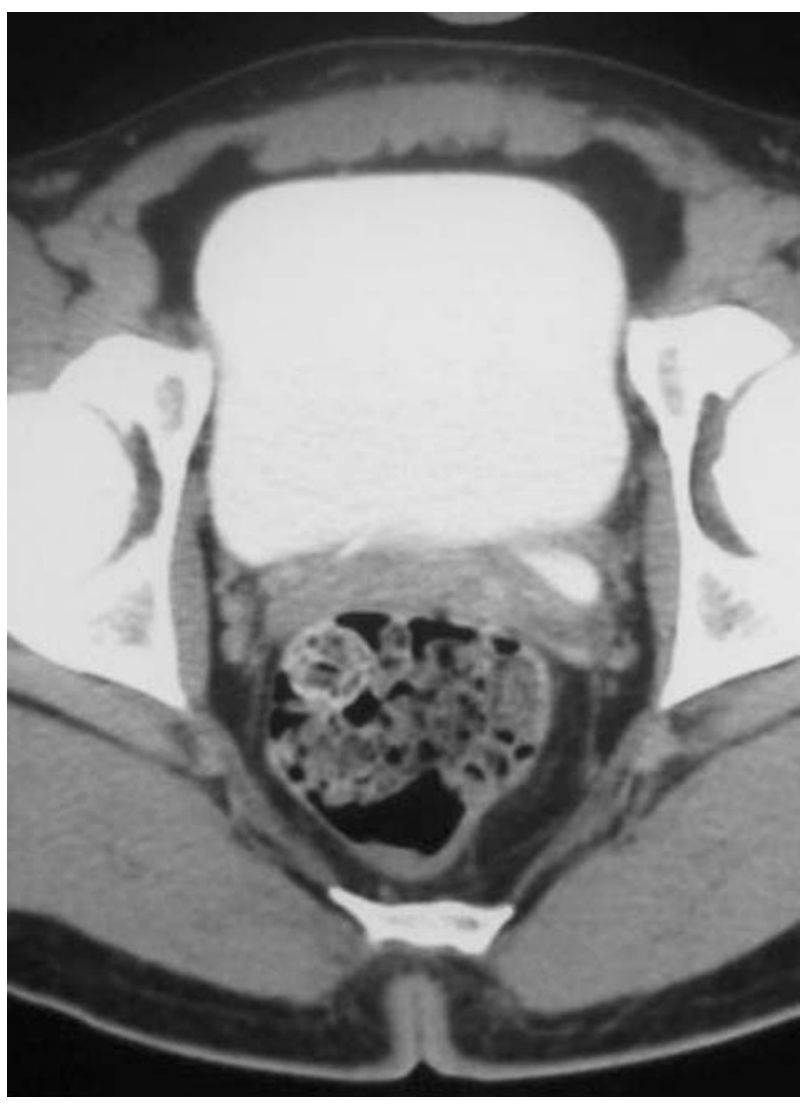

FIGURA 5. Imagen TC postoperatoria del caso la figura anterior. Obsérvese la retracción prácticamente completa tras la incisión transuretral del quiste.

\section{DISCUSION}

Los quistes de la vesícula seminal, aunque de forma infrecuente pueden tener un origen adquirido probablemente relacionado con una obstrucción del conducto eyaculador ${ }^{5}$, la inmensa mayoría de ellos son de origen congénito como consecuencia de una anomalía en el desarrollo del conducto de Wolf, soliéndose asociar a displasia o agenesia renal y en ocasiones, aunque mas infrecuentemente, con abocamiento ureteral ectópico ipsilateral ${ }^{6,7}$. Así, en los casos que presentamos en el presente estudio, en 5 pacientes (62\%) se halló agenesia renal y en 3 casos (38\%), displasia renal y uréter ectópico ipsilateral, 2 de ellos con importante dilatación del remanente ureteral. 
Desde el punto de vista embriológico, la íntima relación en el desarrollo de los sistemas urinario y reproductor podría explicar la coexistencia de dichas anomalías, seminal y renal; pues de la porción mas caudal del conducto mesonéfrico se formarán entre la $4^{a}$ y $13^{\text {a }}$ semanas de gestación una serie de estructuras que darán lugar entre otros al uréter, el conducto deferente, las vesículas seminales y la próstata.

Se han establecido diversas posibilidades o hipótesis para explicar las anomalías congénitas derivadas de dichas estructuras de origen común: a) la ausencia de riñón, uréter, hemitrígono, vesículas seminales y conducto deferente resultaría por un fallo completo del conducto mesonéfrico; b) si únicamente ocurriera un fallo en el desarrollo del brote ureteral, daría como resultado una agenesia renal con una vesícula seminal normal; c) el desarrollo anormal del conducto mesonéfrico distal únicamente, produciría un quiste de vesícula seminal con presencia de riñón normal; y d) el anormal desarrollo de la porción distal del conducto mesonéfrico provocará asimismo un fallo en el brote ureteral, dando lugar a una ausencia renal asociada a una atresia del conducto eyaculador, apareciendo consecuentemente una dilatación quística de la vesícula seminal ${ }^{8-10}$.

Respecto a las manifestaciones clínicas, en muchos casos permanecerá silente durante toda la vida y su diagnóstico constituye un hallazgo casual durante el estudio de otra patología como ocurrió en 3 de los casos que presentamos. Cuando se manifiestan clínicamente, auque pueden hacerlo a cualquier edad, por lo general lo hacen durante la $2^{\underline{a}}$ y $3 \underline{\text { a décadas de }}$ la vida, cuando aumenta la actividad sexual y las secreciones del tracto reproductor causan distensión de la vesícula seminal y los síntomas se producen.

La sintomatología provocada por esta entidad recogida en la literatura es sumamente variada, pudiendo dar lugar a síntomas miccionales tanto obstructivos como irritativos, molestias perineales y abdominales inespecíficas secundarias a la compresión producida por la distensión del quiste así como alteraciones eyaculatorias e infertilidad. No obstante, los síntomas más frecuentemente referidos en diversas publicaciones son: disuria, frecuencia, dolor perineal, epididimitis, dolor posteyaculatorio y dolor del contenido escrotal ${ }^{9}$.

La ecografía, debido a su nula invasividad y alta disponibilidad en la mayor parte de servicios, así como por su bajo coste económico, representa una exploración complementaria insustituible tanto para la valoración inicial que permita realizar el diagnóstico de presunción de esta entidad como para el control evolutivo de los pacientes. Así, en todos nuestros casos, el hallazgo ecográfico de una estructura hipoecogénica de localización retrovesical en la zona de la vesícula seminal junto con la no visualización de la unidad renal homolateral o bien la dilatación de la misma, nos sugirió el diagnostico de esta infrecuente anomalía. Asimismo, la ecografía transrectal, además de ser una exploración de gran valor para el diagnóstico diferencial de la patología quística pélvica, permite la punción-aspiración ecodirigida del quiste, ya sea con fines diagnósticos o terapéuti$\cos ^{11-13}$.

En la urografia intravenosa es casi constante la anulación funcional de la unidad renal homolateral, siendo excepcional su normalidad, debiendo hacernos cuestionar el diagnóstico en tal caso. Además, en la fase cistográfica puede apreciarse un defecto de repleción de contraste ocasionado por la compresión vesical de la vesícula seminal quística. Así, en 5 de los pacientes de este estudio en los que realizamos dicha exploración se puso de manifiesto la anulación funcional renal unilateral y sólo en tres de ellos se observó la alteración en la cistografía.

La deferentovesiculografía, antaño considerada una exploración trascendental y practicada en muchos de los primeros casos descritos, la creemos actualmente injustificada tras la aparición de nuevas técnicas de diagnóstico por imagen ${ }^{9,14}$. Tampoco la práctica de arteriografia para establecer el diagnóstico de agenesia renal, como se sugiere en algunas publicaciones ${ }^{1,6}$, la consideramos actualmente justificada. Igualmente, aunque la cistoscopia puede evidenciar la ausencia de un hemitrígono o la compresión del mismo por el quiste, tampoco la creemos una exploración imprescindible para el diagnóstico.

El análisis del contenido quístico, obtenido mediante punción-aspiración, puede ser de utili- 
dad para el diagnóstico diferencial con otros quistes pélvicos profundos, siendo frecuente el hallazgo de espermatozoides en la entidad que nos ocupa ${ }^{1,4}$.

Después de la ecografia, tanto la TC como la RM constituyen en la actualidad las exploraciones habitualmente utilizadas, ya que permiten definir con mayor precisión las características del quiste, su posible relación con un uréter ectópico, así como el resto de posibles anomalías asociadas $^{9,12}$, y aunque varios autores revisados concluyen que la RM es la mejor prueba de imagen para obtener un claro diagnóstico prequirúrgico de forma no invasiva y que debe ser de elección ante la sospecha de ésta compleja patología $^{11,15}$, creemos que las nuevas generaciones de TC volumétrico también ofrecen una excelente seguridad diagnóstica.

Respecto al tratamiento, es una opinión unánime de todos los autores revisados y así lo confirma nuestra propia experiencia, que en los casos asintomáticos de diagnóstico incidental, la abstención quirúrgica y el seguimiento evolutivo constituye la conducta más adecuada, reservando el tratamiento quirúrgico para los casos sintomáticos.

La punción-aspiración del quiste suele asociarse a resultados poco satisfactorios, ya que la recidiva del contenido quístico es la norma, dando lugar a un elevado índice de recurrencias; y aunque se trata de una técnica mínimamente invasiva, ya sea por vía perineal, transrectal o transabdominal, no está exenta de complicaciones, siendo frecuente la infección del quiste que dificulta posteriormente la cirugía $9,11,13,14$.

La incisión endoscópica transuretral de la vesícula seminal con asa de Collins, drenando el contenido del quiste al interior de la vejiga, representa otra alternativa terapéutica de escasa complejidad y mínima morbilidad utilizada por algunos autores ${ }^{16,17}$ y que nosotros hemos empleado con éxito en tres de nuestros pacientes, obteniendo en todos ellos unos excelentes resultados, invirtiendo tiempos operatorios reducidos así como una rápida recuperación postoperatoria con alta hospitalaria a las pocas horas de la intervención. No obstante, nuestros resultados se oponen a la idea sugerida por otros autores que afirman que el tratamiento de elección lo constituye la exéresis de la vesícula patológica y del remanente renoureteral en caso de existir, aduciendo malos resultados del tratamiento endoscópico como consecuencia de la frecuente aparición de residuo postmiccional por la presencia de un pseudodivertículo formado por la pared del quiste. Sin embargo, en nuestra experiencia, en los tres pacientes tratados endoscópicamente constatamos la retracción prácticamente completa de la vesícula seminal afecta, sin evidenciarse la presencia de pseudodivertículo sintomático (Fig. 5). Así, en los casos que se acompañan de un uréter ectópico dilatado, para evitar la aparición de esta complicación, creemos indicada la exéresis quirúrgica, en la cual, aunque la vía laparoscópica ha sido recomendada por algunos autores con experiencia aportando una reducida morbilidad quirúrgica ${ }^{18,19}$, la mayoría de grupos hemos utilizado la resección en bloque por abordaje retroperitoneal.

\section{CONCLUSIONES}

La ecografía representa una exploración complementaria insustituible, tanto para la valoración inicial que permita realizar el diagnóstico de presunción de esta entidad como para el control evolutivo de los pacientes.

Tanto la TC como la RM permiten habitualmente confirmar el diagnóstico, definiendo con mayor precisión las características del quiste, su posible relación con un uréter ectópico, así como el resto de posibles anomalías asociadas.

En los casos asintomáticos de diagnóstico incidental, la abstención quirúrgica y el seguimiento evolutivo constituye la conducta más adecuada, reservando el tratamiento quirúrgico para los casos sintomáticos.

La incisión transvesical del quiste de la vesícula seminal es un procedimiento mínimamente invasivo y con escasa morbilidad, habiendo constatado en nuestra experiencia la retracción prácticamente completa de la vesícula seminal. Así, la consideramos de elección para el tratamiento de los pacientes afectos de dicha entidad, reservando la exéresis para los casos en los que fracase la técnica por aparición de un pseudodivertículo sintomático o los que presenten ureterohidronefrosis asociada. 


\section{REFERENCIAS}

1. Soler JM, Domínguez C, Cabello J, Murillo J, Torrubia FJ. Quiste de vesícula seminal y agenesia renal ipsilateral: asociación frecuente. Actas Urol Esp 1992;16:800-804.

2. Sheih CP, Hung CS, Wei CF. Cystic dilatations within the pelvis in patients ipsilateral renal agenesis of displasia. $\mathrm{J}$ Urol 1990;144:324-327.

3. Muñoz D, García-Miralles R, Benejam JM. Agenesia unilateral de las estructuras dependientes del conducto mesonéfrico. Presentación de un caso. Actas Urol Esp 2003; 27:312-316.

4. Tramoyeres A, Canovas JA, Sánchez F, De La Torre L, Ramada F, Navalón, P et al. Quiste de vesícula seminal. A propósito de un caso y revisión de la literatura. Arch Esp Urol 2004;57:165-168.

5. Sandlow JI, Williams RD. Cirugía de las vesículas seminales. En: Walls CD editor. Campbell Urologia. $8^{a}$ Ed. Buenos Aires. Panamericana 2004;4235-4252.

6. Dominguez M, Amaya J, Salazar R, Rico J, Camacho E, García M. Displasia quística en vesícula seminal. Caso clínico y revisión de la literatura. Actas Urol Esp 1999;23: 532-535.

7. Ritckey ML, Benson RC, Kramer SA, Kelalis PP. Management of Mullerian duct remnants in the male patient. J Urol 1998;140:795-799.

8. Soler JM, Domínguez C, Herrera J, Murillo J, Castaño JL, Poveda A. Uréter ectópico en vesícula seminal con displasia quística. Caso clínico y revisión de la literatura. Actas Urol Esp 1990;14:447-450.

9. Van Den Ouden D, Blom JH, Bangma C, Spiegeleer A. Diagnosis and management of seminal vesicle cyst associated with ipsilateral renal agenesis: a pooled analysis of 52 cases. Eur Urol 1998;33:433-440.

10. Pellice C, Pares ME, Bassa P. Agenesia unilateral deferencial. Asociación conjunta a una ausencia de epidídimo, vesícula seminal y unidad renal del mismo lado. Caso sucinto. Actas Urol Esp 1998;22:454-457.
11. Pascual M, Egea J, Cortiñas JR, Calleja J, Rivero MD, Fernández E. Agenesia renal derecha y abocamiento ectópico del uréter en dilatación quística de la vesícula seminal. Actas Urol Esp 2004;28:688-693.

12. Berne JM, Bono A, Roncales AL. Desembocadura de uréter ectópico retroiliaco en vesícula seminal. Arch Esp Urol 2001;54:61-64.

13. Negrín A, Arteaga F, Masot JM, Gutiérrez P, Rodríguez JM, Ravina M, et al. Quiste de vesícula seminal. Importancia de la ecografía transrectal en este tipo de malformaciones. Actas Urol Esp 1993;17:132-134.

14. Madrid FJ, Madroñero C, Rivas JA, Parra L, Monsalve M, García J. Quiste de vesícula seminal y agenesia renal ipsilateral. Arch Esp Urol 2004;57:168-171.

15. Roberts SG, García JM, Segura JW. Incidental pelvis mass identified during ultrasound-guided transrectal needle biopsy of the prostate. Arch Esp Urol 2002;49:139-141.

16. Gonzalez CM, Dalton D. Endoscopic incision of a seminal vesicle cyst. Urology 1998;51:831-832.

17. Okoye BO, Jones DJ, Lancashire MJ, Brown EF, Ritchie AW. Transvesical endoscopic drainage of a seminal vesicle cyst. Br J Urol 1995;76:810-811.

18. Ikari O, Castilho LN, Lucena R: Laparoscopic excision of seminal vesicle cyst. J Urol 1999;162:498-501.

19. Cherulo EE, Meraney AM, Bernstein LH. Laparoscopic management of congenital seminal vesicle cyst associated with ipsilateral renal agenesis. J Urol 2002;167:12631267.

Dr. P. Navalón Verdejo

E-mail: pedronavalon@yahoo.com

(Trabajo recibido el 8 de septiembre 2006) 